\title{
Motivación y Valores relativos al Trabajo en Bomberos Voluntarios y Remunerados argentinos
}

\author{
Work motivation and values in Argentine volunteer and paid firefighters
}

\author{
Florencia Rocío Piedimontea, Pablo Domingo Depaula, \\ aUniversidad Abierta Interamericana, Facultad de Psicología y Relaciones Humanas, Argentina

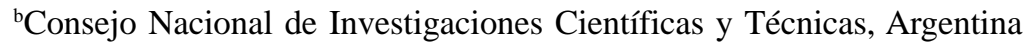 \\ 'Instituto de Investigaciones en Psicología de la Universidad de Buenos Aires, Argentina
}

\section{Resumen}

El Cuerpo de Bomberos es una fuerza de protección para la ciudadanía de un país que por su rol y función, reviste vital importancia para la comunidad. Este estudio indaga la relación entre la Motivación, conceptualizada como una variable orientadora de la conducta y los Valores relativos al Trabajo, considerados como guías en la vida laboral de los bomberos de la Ciudad Autónoma de Buenos Aires (Argentina). Se realizó un estudio descriptivo, correlacional y de diferencia de grupos, cuya muestra estuvo integrada por 130 bomberos (25 mujeres y 105 varones), de los cuales 67 eran económicamente remunerados y 63 eran voluntarios. La edad promedio fue de 32.32 años. Se administró la Escala de Motivación en el Trabajo (adaptación al idioma español de Gagné et al., 2012) y la Escala Revisada de Valores relativos al Trabajo de Porto y Pilati (2010, adaptación argentina de Moreno \& Marcaccio, 2012). Se halló que los bomberos motivados extrínsecamente atribuyen de modo autónomo y deliberado significados a sus acciones basándose en valores de logro personal, en la propia autopercepción de competencia y, sobre todo, de utilidad social (i.e., Altruismo), por lo que su labor resulta acorde a sus expectativas de bienestar personal, y a la vez procura mejorar la vida de sus pares sociales.

Palabras clave: bomberos voluntarios, bomberos remunerados, motivación, valores relativos al trabajo.

Para citar este artículo:

Piedimonte, F., \& Depaula, P. D. (2018). Motivación y valores relativos al trabajo en bomberos voluntarios y remunerados argentinos. Liberabit, 24(2), 277-294. https://doi.org/10.24265/liberabit.2018.v24n2.08

\begin{abstract}
The Fire Department is a protection force for the citizens of a country. Because of its role and function, it is of vital importance to the community. This study investigates the relationship between Motivation, conceptualized as a guiding variable of behavior, and Work Values, considered as guides in the career of firefighters of the Autonomous City of Buenos Aires (Argentina). A descriptive, correlational and group difference study was carried out with a sample of 130 firefighters (25 women and 105 men), of whom 67 were paid and 63 were volunteers. The average age was 32.32 years. The Work Motivation Scale (adapted to Spanish by Gagné et al., 2012) and the Revised Scale of Work Values by Porto and Pilati (2010, Argentine adaptation by Moreno and Marcaccio, 2012) were used. It was found that extrinsically-motivated firefighters confer, autonomously and deliberately, significance to their actions based on values such as personal achievement, self-perceived competence and, above all, social utility (i.e., Altruism). Consequently, their work complies with their expectations of personal wellbeing and, at the same time, is aimed at improving the life of their social peers.
\end{abstract}

Keywords: volunteer firefighters, paid firefighters, motivation, work values.

Este es un artículo Open Access bajo la licencia Creative Commons Atribución-NoComercial-CompartirIgual 4.0

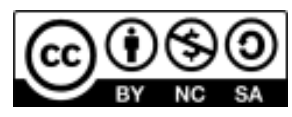




\section{Introducción}

La vida de las personas y las comunidades se encuentran siempre expuestas a situaciones que pueden resultar traumáticas, y que amenazan o ponen en riesgo su integridad psicofísica, pero a veces esas amenazas resultan mayores a la posibilidad de los individuos para afrontarlas; es entonces cuando se requiere de medios extraordinarios (Uriarte-Arciniega, 2010). Dichos «medios extraordinarios» son, entre otros cuerpos de asistencia: los bomberos, una fuerza de protección urbana comunitaria que participa activamente frente a situaciones de emergencia y catástrofe (e.g., incendios, derrumbes e inundaciones).

En la Ciudad Autónoma de Buenos Aires (Argentina), fueron de público conocimiento numerosas intervenciones que han puesto en evidencia el riesgo vital, impacto emocional y «sacrificio» cotidiano que implica la labor bomberil. En este sentido, Calandrón y Galar (2014) expresan que en la comunidad de bomberos (incluyendo a sus familias y también a sus vecinos), «la rutina laboral se describe como una constante entrega con el objetivo de salvar vidas y bienes de personas que son desconocidas para el bombero que interviene». (p. 9).

En tal contexto, resulta necesario conocer bajo qué convicciones se encuentra guiada u orientada la vida laboral de los bomberos. La actividad laboral de cada sujeto, se encuentra vinculada a la búsqueda de metas que para ellos sean significativas. En línea con lo anterior, para comprender qué es significativo o importante para las personas en relación al escenario laboral, se requiere estudiar los valores relativos al trabajo, en tanto representarían metas que guían los intereses y acciones que enmarcan la vida laboral de las personas en diversos ámbitos (i.e., profesional, artístico, militar, entre otros) tal como lo señalan diversos autores (Castro, 2005; Casullo \& Castro Solano, 2004; Depaula, 2012; Depaula \& Azzollini, 2012; Moreno \& Marcaccio, 2012; Schwartz, 1994; Schwartz et al., 2001).
Investigar acerca de los tipos de motivación y valores relativos al trabajo que guían la conducta y vida laboral de los bomberos, permite poder evaluar la necesidad de crear programas y organismos que promuevan el enrolamiento al mencionado cuerpo de asistencia, tomando en consideración los tipos de motivación y valores relativos al trabajo que se evidencien, entre otros factores que puedan influir y de esta forma orientar en la captación de un mayor número de integrantes que nutran el cuerpo de bomberos de la Ciudad Autónoma de Buenos Aires. Este aumento de dotación no solo favorecería la cobertura asistencial para toda la comunidad, sino que además incrementaría el bienestar de los bomberos, quienes realizan jornadas de guardias con extensa carga horaria, que les impide tener un descanso adecuado.

En relación al estudio de la motivación humana, la Teoría de la Autodeterminación (en adelante TAD) elaborada por Ryan y Deci (2000) resulta un constructo de gran relevancia teórica y aplicación empírica para el abordaje del presente estudio; dicha teoría se enfoca en el crecimiento de las personas y sus necesidades psicológicas innatas. Partiendo de procesos empíricos, se han reconocido tres necesidades psicológicas básicas: de competencia, de autonomía, y de relacionarse. Estas deben ser satisfechas durante todo el transcurso de la vida para que un sujeto advierta un sentido continuo de integridad y bienestar, pero si no son satisfechas, conducen a la patología y al malestar. Los estudios desarrollados por la TAD se centran sobre las consecuencias de la medida en la que los individuos son capaces de satisfacer dichas necesidades dentro de los entornos sociales (Ryan \& Deci, 2000).

Gagné y Deci (2005) explican que para la TAD es de suma importancia discernir entre motivación autónoma o autodeterminada y motivación controlada. Revelan que la autonomía supone que el actuar del sujeto esté forjado en la conducta volitiva y la experiencia de elección. En cambio, si se trata de una motivación controlada, esto responde a un accionar signado por la presión (sobre todo externa), y 
acompañado por la sensación de deber participar de cierta tarea.

La TAD desarrolla tres tipos generales de motivación dependiendo del nivel de autodeterminación que posea una persona; distingue entonces: motivación intrínseca, motivación extrínseca y desmotivación (Ryan, 1995).

La motivación intrínseca es la dimensión más autodeterminada de este constructo y se define como la realización de una conducta solo por el placer y la satisfacción derivada de su ejecución (Battistelli, Galletta, Odoardi, Núñez, \& Ntalianis, 2015). Aunque los factores de autonomía y competencia son muy importantes para generar la versatilidad en la motivación intrínseca, un tercer factor, el de relacionarse, también asiste a su expresión. Para la TAD existe una mayor tendencia al desarrollo de la motivación intrínseca en contextos signados por una sensación de seguridad y de estar relacionado (Ryan \& Deci, 2000).

Por otro lado, Battistelli et al. (2015) describen que la motivación extrínseca revela la realización de una tarea por los incentivos o consecuencias asociadas a ella. En línea con la TAD, Deci y Ryan (1995) proponen diferentes tipos de motivaciones extrínsecas que indican distintos grados en los que el valor de la conducta y su regulación han sido internalizados e integrados. La internalización muestra que todas las personas albergan un valor o regulación (Ryan \& Deci, 2000). De esta forma, las conductas extrínsecamente motivadas que son menos autónomas se describen como reguladas externamente. La regulación externa implica que el comportamiento se acciona con el fin de satisfacer una demanda externa, una recompensa, o de evitar castigos (Battistelli et al., 2015; Ryan \& Deci, 2000).

La internalización y la integración son factores claves de la socialización durante la niñez, pero también son importantes para la regulación de la conducta a lo largo de toda la vida. En la gran mayoría de las situaciones que involucren a una persona, estarán dispuestas ciertas conductas que no resulten atractivas y valores que no se adquieran naturalmente. Bajo estas premisas, la TAD ha abordado los factores de las dinámicas mediante las cuales las conductas nointrínsecamente motivadas pueden pasar a ser autodeterminadas, y los modos en los que el entorno social influye en estas dinámicas (Ryan \& Deci, 2000).

Otro tipo de motivación extrínseca es la llamada regulación introyectada. La introyección refiere al hecho de introducir dentro de sí la regulación, pero no admitirla como algo de uno mismo (Ryan \& Deci, 2000). Así, los comportamientos son ejecutados para evitar la culpa o enriquecer el ego (Gagné \& Deci, 2005). Asimismo, otra forma de motivación extrínseca es la regulación identificada que sugiere el hecho de asignarle un valor consciente a una acción o conducta, porque la persona se identifica con el valor o el significado de un comportamiento y lo asume como algo personal importante (Battistelli et al., 2015).

La desmotivación refleja la ausencia de motivación hacia una actividad (Battistelli et al., 2015). Es un estado en el cual la persona carece de la intención de actuar. Es definida también como la consecuencia de no asignarle valor a una actividad (Ryan, 1995), el no sentirse competente para su ejecución, o el no esperar que esta genere un resultado deseado (Bandura, 1975, 1999, 2002).

En relación a los valores humanos dirigidos hacia el trabajo, Porto y Tamayo (2003) los definen como aquellas creencias o fundamentos organizados jerárquicamente que posee una persona, sobre los propósitos o recompensas que desea. Dichos principios se buscan mediante el desarrollo de actividades laborales que orientan la conducta, las evaluaciones sobre sus resultados, el contexto y la elección de opciones laborales.

En la presente investigación se ha considerado el modelo de valores propuestos por Moreno y Marcaccio (2012), que han obtenido como resultado de la adaptación y validación para población argentina de la Escala de Valores relativos al Trabajo de Porto y Pilati (2010); a saber: 
- Realización Personal. Hace alusión al sentimiento de tener experiencias en el trabajo que facilitan el desarrollo de las competencias individuales. Son personas que buscan desenvolverse en lo personal.

- Altruismo. Se vincula al hecho de que una persona se sienta útil para la sociedad y promueva el bien común, aún en detrimento del propio beneficio. Son sujetos que necesitan vivenciar que su trabajo mejora la vida de otras personas.

- Reconocimiento Social. Refiere a la experiencia de sentirse reconocido por la sociedad, ser famoso o admirado por el trabajo.

- Aspiraciones Económicas. Guarda relación con el logro de la independencia financiera. Son personas que procuran a través de su trabajo y de logros materiales mejorar sus condiciones de vida.

Con todo, la temática seleccionada para esta investigación, se encuadra dentro de las variables psicológicas de Motivación y Valores relativos al Trabajo en Bomberos Voluntarios y Bomberos Remunerados de la Ciudad Autónoma de Buenos Aires (Argentina). En tal sentido, la problemática que se aborda surge de la reflexión crítica acerca de la importancia que tiene la función y el rol de los bomberos en la sociedad, al tiempo que la complejidad psicosocial que se entrama al riesgo e incertidumbre ligados a la ocurrencia de accidentes, desastres o catástrofes y a los niveles de vulnerabilidad social de las comunidades potencialmente afectadas (Natenzon \& González, 2010) legitima analizar los valores y motivaciones bomberiles conforme a sus condiciones laborales.

\section{Antecedentes de investigación en motivación y valores en diversidad de voluntarios y profesionales remunerados}

La población de bomberos ha sido objeto de diversas investigaciones en relación al estudio de la motivación, los valores, entre otras variables de interés. En la anterior década, Peñacoba-Puente, Díaz-
Gutiérrez, Goiri-Pueyo y Vega-López (2000) estudiaron las estrategias de afrontamiento utilizadas por bomberos con experiencia y sin experiencia en diferentes situaciones de estrés al analizar intergrupalmente a dos grupos de 30 bomberos de la Comunidad de Madrid. Los resultados reflejaron que los bomberos sin experiencia utilizaban, en mayor medida, estrategias centradas en el análisis lógico, la reevaluación positiva y la evitación cognitiva, inclinándose por consultar a sus superiores al momento de buscar información, guía y apoyo.

Paralelamente, Chacón y Dávila (2001) analizaron diferencias y similitudes entre los perfiles motivacionales de sujetos que realizaban actividades ecologistas y sujetos que realizaban actividades socioasistenciales. La muestra estuvo integrada por 323 sujetos (178 voluntarios que desarrollaban actividades de campo vinculadas a la protección de la naturaleza y 145 voluntarios los cuales desarrollaban actividades socio-asistenciales). Los resultados mostraron diferencias significativas en las motivaciones en función del tipo de actividad de los voluntarios, y un efecto de interacción entre la edad, el género y el tipo de actividad que modulaba las diferencias halladas. Los voluntarios que realizaban actividades de campo para la defensa medioambiental estuvieron más motivados que los socio-asistenciales por la necesidad de expresar valores personales relacionados con un interés hacia la naturaleza. En cambio, los voluntarios que realizaban actividades socio-asistenciales estuvieron más motivados por la necesidad de adquirir nuevas experiencias y tener la oportunidad de ejercitar conocimientos y habilidades. Sin embargo, a pesar del perfil motivacional diferencial encontrado, no se evidenciaron diferencias significativas en la motivación general presentada por los voluntarios. En los voluntarios ambientales, al igual que en los socioasistenciales, coexistieron una gran diversidad de motivos y, de la misma forma, los autores coincidieron en dar más importancia a las motivaciones heterocentradas (i.e., valores humanos), lo que justifica la realización de estudios vinculantes. 
Un estudio realizado por Baard, Deci y Ryan (2004), sobre una muestra de 59 empleados del centro de operaciones de una importante corporación bancaria de EE.UU., indicó que los gerentes que ejercían su rol apoyando la autonomía de sus seguidores, tenían empleados a cargo que experimentaban una mayor satisfacción de las necesidades psicológicas básicas, un mayor compromiso en su labor, evidenciaban buenos niveles de bienestar psicológico, y tenían puntuaciones más altas en su rendimiento, en comparación con los empleados de los gerentes quienes ejercían su rol con un mayor control sobre sus seguidores.

En relación a la temática relativa a los valores en diversos grupos profesionales, Nader y Castro-Solano (2007) realizaron una investigación en Argentina cuya muestra estuvo integrada por 224 líderes que residían en la Ciudad Autónoma de Buenos Aires y en el Conurbano Bonaerense, y que ejercían su labor en pequeñas, medianas y grandes empresas. Considerando el modelo de liderazgo Transformacional/Transaccional de Bass (1990) y la Teoría de los Valores Universales de Schwartz (1994, 2001), los autores analizaron la influencia que ejercían los valores de los líderes sobre sus estilos de liderazgo lo que les permitió observar que los valores de autotrascendencia (i.e., Universalismo y Benevolencia) y de apertura al cambio (i.e., Hedonismo, Autodirección y Estimulación) predecían el estilo de liderazgo Transformacional, i.e., líderes carismáticos que promueven el cambio y la innovación motivando a sus seguidores a trascender sus objetivos personales, promoviendo la cohesión del grupo. Por otro lado, los valores de Autopromoción (i.e., Logro y Poder) fueron predictores del estilo de liderazgo Transaccional, i.e., líderes que negocian con sus seguidores intercambios de promesas y favores para conseguir los objetivos de la organización (Castro, 2005).

Más adelante, Depaula y Azzollini (2012) analizaron la existencia de relaciones entre los niveles de inteligencia cultural, la motivación para el aprendizaje académico y los valores humanos vinculados al trabajo en estudiantes militares argentinos, evaluando una muestra de cadetes aspirantes a Oficiales del Ejército Argentino oriundos de diferentes provincias argentinas. Los resultados del análisis revelaron que los estudiantes portaban valores relacionados en mayor medida al cuidado de las tradiciones nacionales, mientras que el valor Estimulación (Castro, 2005; Schwartz, 1994, 2001) se vinculaba con la resolución creativa y original de experiencias nuevas o situaciones inciertas, equilibrando cierta autorestricción que dificultaría la apertura hacia la diversidad cultural.

Empleando la Escala de Valores Relativos al Trabajo que adaptaron en Argentina en el año 2012, Moreno y Marcaccio (2014) analizaron la relación entre los valores relativos al trabajo y algunos perfiles profesionales (i.e., Contabilidad y Administración, Marketing y Ventas, y Recepción). La muestra estuvo integrada por 425 sujetos los cuales se encontraban buscando un cambio de empleo o un nuevo empleo. Los resultados indicaron que el valor de Realización Personal fue más jerarquizado por los individuos con perfil profesional de Marketing; no se hallaron diferencias según sexo, ni interacción sexo-ocupación, al tiempo que en relación a las subescalas Reconocimiento Social, Altruismo y Aspiraciones Económicas, tampoco se encontraron diferencias.

Por su parte, Gagné et al. (2014) seleccionaron una muestra de 3435 trabajadores a lo largo de nueve países y con siete idiomas diferentes, a fin de realizar la validación de una escala para evaluar la Motivación en el Trabajo. Los autores concluyeron que las necesidades psicológicas de autonomía, de competencia y de relación, previsiblemente se relacionaban con las diferentes formas de motivación propuestas por la Teoría de la Autodeterminación (ya descrita anteriormente), mientras que la motivación autónoma se relacionaba positivamente con el funcionamiento óptimo de los individuos (e.g., el bienestar, el rendimiento), siendo la motivación controlada y menos beneficiosa. 
Battistelli et al. (2015) crearon una versión abreviada de la Escala de Motivación en el Trabajo (MWMS) analizando una muestra de 1035 trabajadores de Grecia, Italia y España. Los resultados confirmaron que las formas intrínsecas e integradas de la Motivación Autónoma (conforme a la Teoría de la Autodeterminación) tuvieron una relación positiva con la satisfacción laboral, las relaciones afectivas, el compromiso con el trabajo, la conducta prosocial, el apoyo organizativo percibido, la autonomía en el trabajo, y el intercambio líder-miembro. En cambio, las formas controladas de motivación (en su aspecto social y material) no se encontraron relacionadas con la mayoría de las variables examinadas, a excepción de la satisfacción laboral con la cual la Motivación Controlada se asoció negativamente, y el compromiso de continuidad con la cual se asoció positivamente. Por último, la Desmotivación se relacionó negativamente con todas las variables vinculadas con los empleados, a excepción del compromiso de continuidad.

En relación a la cuestión vinculada con la satisfacción ocupacional de los bomberos, un estudio realizado en Canadá por Wagner y O’Neill (2012) arrojó resultados con algunas implicancias para el presente estudio. Los autores mencionados seleccionaron un grupo experimental de 119 miembros del departamento de bomberos del Norte de la provincia de Columbia Británica, de sexo masculino en su totalidad, que incluía 11 sujetos que integraban el staff administrativo, 11 empleados del call center correspondiente a la línea de emergencia «911» y 97 bomberos profesionales de tiempo completo, y un grupo de comparación integrado por 66 trabajadores también de sexo masculino de la misma comunidad canadiense (equitativos sociodemográficamente al grupo experimental en variables tales como la edad, nivel educativo, horario de trabajo, años de servicio y etnia). A la totalidad de la muestra $(N=185)$, se les administró una batería de test psicométricos que incluían el Test de Ajuste Marital de Locke-Wallace (1959), la Escala de Satisfacción con la Vida (SWLS; Diener, Emmons, Larson, \& Griffin, 1985), la
Encuesta de Satisfacción Laboral (Traut, Larsen, \& Feimer, 2000) y, por último, el Inventario de Personalidad Revisado (NEO-PI-R; Costa \& McCrae, 1992) el que evalúa los Cinco Grandes factores de la personalidad (i.e., Responsabilidad o Conciencia, Agradabilidad o Amabilidad, Extraversión, Apertura a la Experiencia y Neuroticismo). Los resultados del estudio indicaron en primer lugar, que los bomberos profesionales presentaban una significativamente mayor satisfacción laboral global que los empleados que integraban el grupo de comparación, al tiempo que fue significativamente menor su satisfacción relacionada al supervisor o jefe directo y a las relaciones departamentales, en comparación con la población general. Asimismo, el factor de la personalidad Responsabilidad o Conciencia constituía un predictor significativamente positivo de la satisfacción laboral global de los bomberos profesionales, mientras que el Neuroticismo predecía negativamente de forma significativa la satisfacción laboral global relacionada con el supervisor o jefe directo. La Satisfacción con la Vida y la cantidad de años de servicio profesional no evidenciaron resultados significativos en ninguna de las dos muestras analizadas.

Por otra parte, un estudio previo en el que se analizó una muestra de 106 voluntarios australianos conformada por 36 preparadores voluntarios de alimentos, 38 preparadores de alimentos con entrega a domicilio empleados en varios hoteles y cafeterías en el área metropolitana de Adelaida (cocineros, aprendices, trabajadores de cocina) y 32 bomberos voluntarios a los que se les administró el Inventario de Personalidad Revisado (NEO-PI-R; Costa \& McCrae, 1992), evidenció correlaciones positivas significativas entre el voluntarismo y los factores de personalidad Extraversión (i.e., expresión de calidez y emociones positivas) y Agradabilidad o Amabilidad (i.e., confianza, altruismo y emociones positivas). Asimismo, un mayor nivel de Extraversión correlacionó positivamente también con un mayor número de horas semanales dedicadas al voluntarismo y con una mayor cantidad de años de servicio 
alcanzados en dicha labor. Los bomberos voluntarios resultaron más asertivos que los preparadores voluntarios de alimentos, lo que para los investigadores puede atribuirse a la naturaleza de la lucha contra incendios, lo que les exige a los bomberos ser más empáticos con las personas a quienes rescatan y disponer de una mayor habilidad social y comunicativa que la preparación de alimentos, especialmente en lo que respecta a las cualidades de liderazgo y la toma de decisiones efectiva que demanda el ámbito bomberil (Elshaug \& Metzer, 2001).

Los estudios comentados podrían presentar algunas implicancias relevantes para la presente investigación en favor de la manera en que variables «psicológicas» como la personalidad modularían la satisfacción por el trabajo profesional realizado, existiendo estudios actuales realizados en Argentina relativos al diseño de un perfil de voluntarios en primeros auxilios psicológicos para población bomberil (i.e., Defensa Civil, Cruz Roja Argentina, etc.), en cuyo caso el factor de la personalidad Apertura a la Experiencia (i.e, personalidades imaginativas, con preferencia por la variedad e intelectualmente curiosas; Castro, 2005) constituye un predictor significativamente positivo de la efectividad alcanzada tras aplicar este tipo de primera respuesta ante situaciones de emergencia o catástrofe. No obstante, más allá de los hallazgos mencionados que vinculan la satisfacción laboral general a una personalidad responsable y ordenada, confiable, con fuerte voluntad y determinación (Castro, 2005) en bomberos profesionales remunerados y, por otro lado, una personalidad extravertida, amable y asertiva asociada a los bomberos que trabajan voluntariamente, se desconocen, hasta el momento, las relaciones entre los valores y motivaciones bomberiles conforme a las retribuciones económicas recibidas (i.e., la actuación del bombero voluntaria o profesionalmente remunerada).

En síntesis, el concepto de motivación ha sido frecuentemente empleado para explicar las razones que dan origen a la conducta de las personas (e.g., Gastañaduy-Fiestas, 2013), en relación a diversidad de actividades y profesiones, al tiempo que el abordaje de los valores humanos de las personas desplegados en el ámbito laboral constituye un área de conocimiento escasamente estudiada en relación al tipo de motivaciones (i.e., de voluntariado o por la obtención de una remuneración, que subyacen a la labor bomberil).

El objetivo general de este trabajo es relacionar los tipos de motivación y valores humanos dirigidos al trabajo en bomberos voluntarios y remunerados de la Ciudad Autónoma de Buenos Aires. Para cumplir con el objetivo señalado se plantearon las siguientes hipótesis:

- H1: Los bomberos remunerados poseen una mayor aspiración económica que los bomberos voluntarios.

- H2: Existe una relación positiva entre la motivación y los valores relativos al trabajo.

\section{Método}

\section{Diseño}

Se realizó un estudio descriptivo, correlacional y de diferencia de grupos (Hernández, Fernández, \& Baptista, 2006).

\section{Participantes}

Se seleccionó una muestra integrada por 130 bomberos (25 mujeres y 105 varones) de los cuales 67 ejercían su rol de forma remunerada (i.e., recibían una paga por su labor) y 63 eran voluntarios (i.e., no recibían ningún tipo de compensación económica por el trabajo realizado). La edad promedio fue de 32.32 años (la edad mínima fue de 14 años y la edad máxima 56 años). Los criterios de inclusión empleados fueron la condición de ser bomberos voluntarios y remunerados con un mínimo de 6 meses en el ejercicio de la profesión dentro de la Ciudad Autónoma de Buenos Aires. Se excluyeron bomberos jubilados, bomberos bajo licencia y bomberos extranjeros. 


\section{Instrumentos}

Escala de Motivación en el Trabajo (R-MAWS). Para medir la Motivación en el Trabajo se utilizó dicha escala, originalmente construida por Gagné et al. (2010), y adaptada luego al idioma español por Gagné et al. (2012). El instrumento ofrece una conceptualización multidimensional de la motivación en el contexto de trabajo, posibilitando la evaluación del tipo y calidad de motivación presente en los sujetos evaluados. Se integra de 19 ítems autoadministrables respondibles a través de una escala tipo Likert que va de 1 (Absolutamente no por esta razón) a 7 (Exactamente por esta razón).

Los 19 ítems de esta versión adaptada y validada al idioma español se obtuvieron realizando un Análisis Factorial Confirmatorio (AFC), del que resultaron cinco factores: Motivación Intrínseca (3 ítems), Regulación Externa (6 ítems), Regulación Identificada (3 ítems), Regulación Introyectada (4 ítems) y Desmotivación (3 ítems).

Los valores obtenidos en el mencionado AFC fueron, para el estadístico, S-B $\chi^{2}=202.670$, con un CFI de .922. El valor correspondiente al error de la media cuadrática RMSEA fue de .060 (indicando un buen ajuste del modelo), mientras que el RMSEA 90\% fue de .040 con un intervalo de confianza de .077 .

Los resultados obtenidos por los autores en relación al cálculo del coeficiente alfa de Cronbach para la escala validada al español revelaron: Regulación Externa ( $\alpha$ $=.78$ ), Regulación Introyectada $(\alpha=.75)$, Regulación Identificada ( $\alpha=$.73), Motivación Intrínseca $(\alpha=.80)$, y Desmotivación $(\alpha=.88)$ (Gagné et al., 2012). Respecto al cálculo del coeficiente alfa de Cronbach del instrumento en esta investigación, los resultados fueron los siguientes: Regulación Externa $(\alpha=.81)$, Regulación Introyectada $(\alpha=.68)$, Regulación Identificada $(\alpha=.76)$, Motivación Intrínseca $(\alpha=.80)$ y Desmotivación ( $\alpha=.67)$, mostrando niveles adecuados o moderados de confiabilidad en las diferentes dimensiones del constructo.
Escala de Valores Relativos al Trabajo (EVT-R). Para evaluar los Valores Humanos dirigidos al Trabajo, se utilizó la Escala Revisada de Valores Relativos al Trabajo de Porto y Pilati (2010), adaptada en Argentina por Moreno y Marcaccio (2012), quienes afirman que representa un instrumento valioso para ser utilizado a lo largo de la vida laboral de las personas (i.e., anticipar, evaluar y mejorar el desempeño laboral, facilitar la toma de decisiones vocacionales, etc.). Se dispone de 20 ítems autoadministrables cuyo continuo de respuesta se instrumenta mediante una escala tipo Likert que va de 1 (Para nada de acuerdo) a 4 (Totalmente de acuerdo).

Con respecto a la validez del instrumento, Moreno y Marcaccio (2012) llevaron a cabo un Análisis de Componentes Principales (ACP) utilizando el método de rotación oblicua con Kaiser. Dicho análisis arrojó una estructura factorial de 20 ítems agrupados en cuatro factores: Realización Personal (5 ítems), Altruismo (5 ítems), Reconocimiento Social (5 ítems) y Aspiraciones Económicas (5 ítems). La medida de adecuación muestral de Kaiser-Mayer-Olkin fue de .861 y el valor obtenido tras realizar la prueba de esfericidad de Bartlett fue de 3418.29 ( $p=.0001)$. Los pesos factoriales de los ítems fueron elevados en todas las escalas (e.g., la carga factorial más baja de los cuatro factores fue de .492). Los autores encontraron dimensiones diferentes a las seis descriptas en la escala original para población brasileña, decidiendo eliminar 18 ítems por complejidad factorial o por baja carga factorial.

Realizado el ACP descrito arriba, los autores calcularon el coeficiente alfa de Cronbach para las cuatro subescalas, obteniendo: Reconocimiento Social $(\alpha=.80)$, Altruismo $(\alpha=.82)$, Aspiraciones Económicas $(\alpha=.79)$ y Realización Personal $(\alpha=$ .76). En cuanto al cálculo del coeficiente alfa de Cronbach del instrumento para el presente trabajo, los resultados fueron: Reconocimiento Social $(\alpha=.78)$, Altruismo $(\alpha=.77)$, Aspiraciones Económicas ( $\alpha=$ .91) y Realización Personal $(\alpha=.80)$, evidenciando niveles adecuados de confiabilidad en la totalidad de las dimensiones escalares. 
Cuestionario Sociodemográfico. Con el propósito de obtener el perfil sociodemográfico de la muestra seleccionada, se diseñó ad hoc un cuestionario de preguntas abiertas y cerradas con alternativas fijas procurando indagar acerca de determinadas variables (e.g., edad, sexo, cargo jerárquico o escalafón).

\section{Procedimiento}

Para recolectar los datos, se administraron conjuntamente la Escala de Motivación en el Trabajo, la Escala de Valores Relativos al Trabajo y el Cuestionario Sociodemográfico, en 11 establecimientos pertenecientes a Bomberos Voluntarios y Remunerados de la Ciudad Autónoma de Buenos Aires.

En primera instancia, se solicitó la autorización institucional a cada establecimiento, explicando a la autoridad a cargo cuál era la finalidad del trabajo, aclarando que posteriormente no se difundirían datos que identifiquen a personas ni a instituciones.

Luego de obtener dicha autorización, se administraron los protocolos de investigación al personal de bomberos de los diferentes turnos.

La batería de test estaba precedida por una consigna que inicialmente invitaba a los colaboradores a participar de una investigación sobre Motivación y Valores relativos al Trabajo en Bomberos Voluntarios y Remunerados; acto seguido, se aclaraba el carácter «voluntario» de los sujetos para responder al protocolo de investigación respectivo. En la misma, también se mencionaba qué variables se iban a estudiar. Además la consigna puntualizaba en el carácter confidencial de la información brindada por el encuestado y se enfatizaba en que los datos obtenidos no serían difundidos y que los resultados serían utilizados únicamente para fines académicos-científicos. Finalmente, se aclaraba que no existían respuestas correctas ni incorrectas y se solicitaba no omitir preguntas, es decir, responder a todo el cuestionario.

Antes de comenzar con la administración de los test, se aclaró nuevamente a los sujetos que serían evaluados que el carácter voluntario de su participación les permitiría decidir interrumpir o abandonar definitivamente la misma (i.e., dejar de responder a las pruebas) en cualquier momento de la administración y ante cualquier circunstancia o eventualidad personal (e.g., incomodidad con el contenido de los reactivos, fatiga o malestar psicológico, o con la temática central de la investigación).

A los fines de formalizar este proceso, los participantes firmaron un «consentimiento informado» ratificando o rectificando su decisión de participar del estudio.

\section{Análisis de datos}

A fin de testear las hipótesis planteadas (conforme al estudio descriptivo, correlacional y diferencial de grupos diseñado), el proceso de análisis de los datos recopilados consistió primeramente en realizar sucesivos análisis de estadística descriptiva (i.e., cálculo de medias y desvíos estándar) de las variables sociodemográficas del estudio (i.e., edad, sexo, y ejercicio del rol de los participantes -bomberos Remunerados vs. Voluntarios-, principalmente).

En segundo lugar, se verificó la normalidad de la muestra a través del Test de Kolmogorov-Smirnov, lo que facilitará posteriormente la decisión metodológica de realizar análisis estadísticos paramétricos o no paramétricos, de acuerdo a la distribución muestral verificada.

Acto seguido, para verificar la H1, i.e., que los Bomberos Remunerados poseían una mayor aspiración económica que los Bomberos Voluntarios, se llevó a cabo la prueba U de Mann-Whittney (U) cumpliendo con el objetivo de comparar dos grupos muestrales con características diferentes en relación al ejercicio de su rol bomberil (i.e., Remunerados vs. Voluntarios).

Para poner a prueba la H2, i.e., la existencia de una relación positiva entre la motivación y los valores 
relativos al trabajo, se llevó a cabo un análisis correlacional utilizando el coeficiente Rho de Spearman $\left(r_{s}\right)$.

Los análisis mencionados fueron realizados mediante el paquete estadístico SPSS 22.0 (versión en castellano), aceptándose valores de significación de $p<.05$ para la interpretación de los resultados arrojados por el proceso de análisis estadístico detallado arriba.

\section{Resultados}

Preliminarmente, para verificar si las muestras respondían a una distribución normal, se realizó la prueba de normalidad a través del Test de Kolmogorov-Smirnov, mostrando una distribución asimétrica de la muestra; es decir que las variables Motivación en el Trabajo y Valores relativos al Trabajo no respondían a una distribución normal $(p<.05)$. Por tanto, se realizaron análisis estadísticos no paramétricos: para diferenciar grupos se utilizó la prueba $U$ de Mann-Whittney (análisis de solo dos grupos), mientras que para el análisis de correlaciones se calculó el coeficiente Rho de Spearman.

\section{Diferencias en Valores Relativos al Trabajo y Motivación en el Trabajo entre Bomberos Voluntarios y Bomberos Remunerados}

El análisis comparativo de ambos grupos de bomberos arrojó diferencias significativas entre la condición de realizar la actividad voluntariamente o recibiendo una paga monetaria. Los Bomberos Remunerados presentaron puntuaciones más elevadas en relación a valores basados en Aspiraciones Económicas ( $U=1431.500 ; p=.001 ; r=.279)$, evidenciando un tamaño de efecto bajo, tendiendo a intermedio (Cohen, 1988). Tal hallazgo indica la presencia de un mayor interés asociado al logro de independencia financiera mediante la actividad laboral que aporte mejoras a las condiciones de la vida personal.

Asimismo, los Bomberos Remunerados evidenciaron puntuaciones más elevadas en la dimensión de Desmotivación $(U=1823.500 ; p=$ $.041 ; r=.18$ ), evidenciando un tamaño de efecto bajo (Cohen, 1988). Dicho resultado indica relativamente la ausencia de significado o incentivo motivacional al momento de realizar una labor, quedando el desarrollo de su actividad sostenida por la paga recibida a cambio (ver Tabla 1).

\section{Correlaciones entre Motivación en el Trabajo y Valores relativos al Trabajo en bomberos}

Primeramente, para analizar la relación entre la Motivación en el Trabajo y los Valores relativos al Trabajo se calculó el coeficiente de correlación, arrojando una asociación positiva y significativa entre la Regulación Externa y el valor Reconocimiento Social $\left(r_{\mathrm{s}}=.287 ; p=.001\right)$, i.e., que un comportamiento es en mayor medida ejecutado por el motivo de obtener una recompensa externa de tipo social, tal como la búsqueda del reconocimiento por parte de la sociedad o bien la búsqueda de prestigio profesional.

Asimismo, la Desmotivación se correlacionó con el valor Reconocimiento Social $\left(r_{s}=.264 ; p=.002\right)$, evidenciando que las personas que carecen de motivación por su trabajo bomberil tienden a buscar también el reconocimiento de sus pares a los fines de poder realizar las tareas que le demanda dicho rol, refugiándose en el sentimiento de que su labor resulta debidamente valorada por la sociedad a la que presta su servicios cotidianamente.

Por otro lado, la Regulación Identificada se asoció con los valores Altruismo $\left(r_{\mathrm{s}}=.379 ; p=.000\right) \mathrm{y}$ Realización Personal $\left(r_{\mathrm{s}}=.433 ; p=.000\right)$, evidenciando que los bomberos motivados extrínsecamente atribuyen de modo autónomo y deliberado significados a sus acciones basándose en el logro personal, la propia autodeterminación o autopercepción de competencia y, sobre todo, de «utilidad social» (i.e., Altruismo), siendo su labor acorde a sus expectativas de bienestar personal, pero a la vez procurando mejorar la vida de sus pares sociales. En suma, un alto nivel de motivación extrínseca dada en términos de regulación identificada 
Tabla 1

Diferencias de rangos de Valores relativos al Trabajo y Motivación en el Trabajo entre Bomberos Voluntarios y Bomberos Remunerados

\begin{tabular}{|c|c|c|c|c|c|c|}
\hline & $\begin{array}{c}\text { Rango Promedio } \\
\text { Bomberos } \\
\text { Remunerados }\end{array}$ & $\begin{array}{c}\text { Rango Promedio } \\
\text { Bomberos } \\
\text { Voluntarios }\end{array}$ & $\begin{array}{l}U \text { de Mann- } \\
\text { Whittney }\end{array}$ & $\mathrm{Z}$ & Sig. & $r$ \\
\hline Reconocimiento Social & 66.67 & 64.40 & 2037.000 & -.344 & .731 & -.030 \\
\hline Altruismo & 69.70 & 61.55 & 1846.000 & -1.241 & .214 & -.109 \\
\hline Aspiraciones Económicas & 76.28 & 55.37 & 1431.500 & -3.179 & .001 & -.279 \\
\hline Realización Personal & 68.09 & 63.07 & 1947.500 & -.766 & .444 & -.067 \\
\hline Regulación Externa & 63.15 & 66.71 & 1962.500 & -.545 & .586 & -.048 \\
\hline Regulación Introyectada & 67.77 & 62.43 & 1905.000 & -.812 & .417 & -.071 \\
\hline Regulación Identificada & 65.93 & 64.11 & 2020.500 & -.279 & .781 & -.024 \\
\hline Motivación Intrínseca & 63.17 & 67.69 & 1964.000 & -.687 & .492 & -.060 \\
\hline Desmotivación & 70.06 & 61.22 & 1823.500 & -2.047 & .041 & -.180 \\
\hline
\end{tabular}

Nota: a. Variable de agrupación: en este cuartel Ud. es bombero voluntario o remunerado.

se asociaría a una marcada necesidad de sentir que la labor bomberil satisface el bien común por encima de los intereses o aspiraciones personales económicas y del reconocimiento o la admiración social.

Por último, la Motivación Intrínseca se asoció con los valores Altruismo $\left(r_{\mathrm{s}}=.369 ; p=.000\right)$, Realización Personal $\left(r_{\mathrm{s}}=.466 ; p=.000\right)$ y con Aspiración Económica $\left(r_{\mathrm{s}}=.175 ; p=.046\right)$, lo que indica comportamientos motivados por el placer inherente a la ejecución de la tarea en sí misma y a la búsqueda de autonomía mediante el desarrollo de competencias individuales y la búsqueda de independencia económica (ver Tabla 2).

Cabe aclarar que los resultados arrojados por el análisis de correlaciones presentados arriba resultan aceptables, en tanto los coeficientes de correlación son cercanos a la «mediana», conforme a la clasificación de tamaños del efecto de Cohen (1992; Meyer et al., 2001).

\section{Discusión}

El presente estudio ha planteado como objetivo general relacionar los tipos de motivación y valores dirigidos hacia el trabajo en bomberos argentinos voluntarios y remunerados de la ciudad capital, cuyos resultados aportan información de relevancia para comprender la dinámica de las expectativas e intereses de personas que realizan tareas cruciales para la protección de la comunidad y la gestión del riesgo.

Analizando la correlación entre las variables de Motivación y Valores dirigidos hacia el Trabajo, se encontraron asociaciones significativas entre sus dimensiones. La Regulación Externa se asoció de forma positiva con el valor Reconocimiento Social, expresando cómo un comportamiento es ejecutado por el motivo de obtener una recompensa externa de tipo social, como puede ser el caso de la búsqueda del reconocimiento por parte de la sociedad o bien la búsqueda de prestigio profesional.

Aquí cabe destacar lo desarrollado por Calandrón y Galar (2014), quienes han realizado un trabajo acerca de la «Elaboración Pública de la Muerte y el Heroísmo en la figura de Bomberos» a partir de la tragedia de Barracas (e.g., incendio de un depósito de documentación en un barrio de la Ciudad Autónoma de Buenos Aires). En dicho estudio, los autores destacan el modo en que el mismo cuerpo de bomberos, sus respectivas familias y hasta los vecinos de las comunas porteñas, describen a la labor bomberil como una acción de entrega y sacrificio cotidiano. Es entonces esperable, que ante la presencia de un 
Tabla 2

Correlación entre Motivación y Valores relativos al Trabajo

\begin{tabular}{llcccc}
\hline & & $\begin{array}{c}\text { Reconocimiento } \\
\text { Social }\end{array}$ & Altruismo & $\begin{array}{c}\text { Aspiraciones } \\
\text { Económicas }\end{array}$ & $\begin{array}{c}\text { Realización } \\
\text { Personal }\end{array}$ \\
\hline \multirow{2}{*}{ Regulación Externa } & Coeficiente de correlación & $.287^{* *}$ & -.065 & .162 & -.046 \\
& Sig. (bilateral) & .001 & .461 & .066 & .608 \\
Regulación Introyectada & Coeficiente de correlación & .096 & .078 & .157 & .161 \\
& Sig. (bilateral) & .278 & .377 & .075 & .069 \\
Regulación Identificada & Coeficiente de correlación & .070 & $.379^{* *}$ & .031 & $.433^{* *}$ \\
& Sig. (bilateral) & .428 & .000 & .726 & .000 \\
Motivación Intrínseca & Coeficiente de correlación & .096 & $.369^{* *}$ & $.175^{*}$ & $.466^{* *}$ \\
& Sig. (bilateral) & .275 & .000 & .046 & .000 \\
Desmotivación & Coeficiente de correlación & $.264^{* *}$ & -.017 & .162 & .077 \\
& Sig. (bilateral) & .002 & .844 & .065 & .386 \\
\hline
\end{tabular}

Nota: **La correlación es significativa al nivel .01 (bilateral). *La correlación es significativa al nivel .05 (bilateral).

comportamiento impulsado por la obtención de recompensas externas (i.e., Regulación Externa), una de las metas que orienta dicho comportamiento sea el valor Reconocimiento Social, a través del cual el sujeto buscará gratificarse mediante la experiencia de sentir que su labor es reconocida o admirada por la sociedad. En consecuencia, este hallazgo tendría algunas implicancias sobre los lazos institucionales que establezcan los cuarteles con los barrios que los rodean, en tanto, el acercamiento mutuo entre bomberos y vecinos (e.g., a fin de año, se ofrece la adquisición de bonos de contribución en cada domicilio, materializados en «rifas» o «sorteos») permite mostrar a la comunidad el interés por reconocerse, a través de un intercambio monetario y a la vez social mínimo que contribuya a mejorar los lazos y necesidades recíprocas. Asimismo, la Desmotivación también correlacionó positivamente con el valor Reconocimiento Social lo que pone en evidencia que la búsqueda de retribución «de la sociedad», de la comunidad, tendería a disminuir la Desmotivación, como alternativa diferente a las compensaciones económicas. A los aportes de Calandrón y Galar (2014), se adicionan las ideas de Arce (2015), quien a partir de una entrevista realizada al personal de bomberos de la Policía Federal Argentina, pone de manifiesto la manera en que el malestar que sufren por la escasez de equipamiento y herramientas necesarias para un correcto desarrollo de la tarea bomberil (situación que podría provocar Desmotivación), es compensado mediante el reconocimiento de los vecinos quienes solidariamente no solo suelen donar recursos materiales (e.g., pintura para reparar los edificios y equipamiento para realizar ejercicio físico), sino que además en fechas festivas, se acercan a los cuarteles ofreciendo regalos en agradecimiento a su labor.

Por otro lado, la Regulación Identificada se relacionó con los valores de Realización Personal y Altruismo. En este caso, los motivos de la acción se vinculan con vivencias de identificación en relación a valores como el desarrollo personal competente y autónomo, y el deseo de ser útil para una comunidad $\mathrm{y}$, de esa forma, a través del trabajo mejorar el bienestar social (Gagné \& Deci, 2005; Moreno \& Marcaccio, 2012). Si se considera entonces la importancia motivacional y de valores humanos vinculados al bienestar social de las comunidades que hacen al contexto de la labor bomberil, dichos resultados tendrían implicancias materializadas en la organización de espacios de desarrollo social, i.e., la creación de comedores comunitarios, la formación de brigadas infantiles para la detección y concientización 
acerca de riesgos cotidianos del hogar, la escuela y la comunidad, centros de alfabetización, de atención a adultos mayores, jubilados y pensionados, talleres y fundaciones de promoción de la inserción social, la solidaridad, y la resiliencia comunitaria (Alchourrón de Paladini, Daverio, Moreno, \& Piattini-Montero, 2011; Melillo, 2011; Ramirez et al., 2013; Scrymgeour, Smith, \& Paton, 2016) de niños en situación de riesgo psicosocial.

Es válido entonces, mencionar el trabajo realizado por Moreno y Schmidt (2013) acerca de las «Motivaciones Altruistas en la elección vocacionalocupacional», en el cual plantearon un interrogante en relación a los motivos que llevan a una persona a elegir determinada profesión vinculada con el servicio dirigido a los demás. En relación a este y a otros cuestionamientos, los autores mencionados compartieron diversas investigaciones, como la de Hernández (2004) quien ha analizado una muestra de 1250 estudiantes españoles de nivel secundario y concluyó que existen diferentes consecuencias o refuerzos esperados en función de sus elecciones vocacionales, distribuidas en cuatro factores: altruismo, seguridad, poder-prestigio e independencia. Asimismo, distinguió dos subgrupos en los cuales el logro de la independencia y el altruismo son "consecuencias intrínsecas» y la seguridad y el poder-prestigio se agrupan como «consecuencias extrínsecas». El autor afirmó que las diversas áreas vocacionales (e.g., enseñanza, sanidad, entre otras) se caracterizan en forma diferencial por el tipo de consecuencias anticipadas. Así, halló una relación entre la anticipación de refuerzos de tipo altruista y la elección de optar por áreas vocacionales tales como «enseñanza», «derecho» y «sanidad». A su vez, también se demostró que las adolescentes mujeres se orientan en mayor medida que los varones a la anticipación de consecuencias «intrínsecas» (i.e., altruismo e independencia). Otro hallazgo destacado por Moreno y Schmidt (2013) fue el obtenido por Soria, Guerra, Giménez y Escanero (2006) quienes encontraron que el motivo fundamental por el que todos los estudiantes, y en especial las mujeres, eligieron la carrera de
Medicina es de carácter altruista-humanitario (i.e., «ayudar a los demás o a la sociedad»), evidenciando un alto grado de vocación social. Finalmente, un estudio realizado en España con estudiantes de la carrera de Medicina (Gutiérrez-Medina, CuencaGómez, \& Álvarez-de Toledo, 2008) mostró que entre las razones que revisten importancia al momento de elegir dicha carrera, se destacaron los motivos altruistas y humanitarios (i.e., necesidad de ayuda, entrega y servicio hacia los demás); en el mismo estudio, otro hallazgo tuvo que ver con que el nivel de este tipo de motivaciones se incrementa considerablemente cuando el alumno comienza a concurrir a hospitales y tiene contacto con pacientes. Tal como se ha mencionado, lo mismo ocurre en la profesión de bomberos, quienes además de expresar sus motivos altruistas y humanitarios mediante la elección de su profesión, a través de la cual buscan la posibilidad de expresar un aspecto de sí mismo que ellos valoran como importante (i.e., Regulación Identificada) probablemente sostengan dicha motivación, debido al contacto que mantienen con los beneficiarios de su ayuda en cada intervención. Dicho contacto reforzaría además la convicción de estar expresando a través de su tarea algo que ellos previamente consideraban un valor importante (i.e., valores relativos al Altruismo).

Continuando con la correlación entre variables, la Motivación Intrínseca se asoció con los valores Altruismo, Realización Personal y Aspiración Económica. Aquí se evidencia un comportamiento motivado por el placer inherente a la ejecución de la tarea en sí y a la búsqueda de autonomía mediante el desarrollo de competencias individuales y a la búsqueda de independencia económica (Ryan \& Deci, 2000; Moreno \& Marcaccio, 2014). En relación a lo mencionado, puede señalarse que en la totalidad de la muestra de bomberos, conviven tanto metas o motivaciones personales laborales intrínsecas como extrínsecas. Puntualmente, la asociación entre la Motivación Intrínseca con los valores de Altruismo, Realización Personal y Aspiración Económica, permite pensar acerca de lo desarrollado por Zubieta y Filippi 
(2007), quienes tomando los señalamientos de Schwartz (2005) indican que «Las circunstancias de vida proveen oportunidades para perseguir o expresar algunos valores más fácilmente que otros o, hacen que la persecución-expresión de diferentes valores sea más o menos costosa o reconfortante» (p. 85). En este sentido, dado que la muestra de bomberos del presente trabajo, si bien impulsa su comportamiento mediante algunos motivos intrínsecos (i.e., por el disfrute inherente al desarrollo de la actividad bomberil en sí misma) buscando incrementar tanto el bienestar de los demás como el propio desarrollo de competencias y habilidades, es atinado considerar sin perjuicio de ello, que las circunstancias de vida (e.g., satisfacción de necesidades fisiológicas básicas) puedan llevar a que también busquen recompensas extrínsecas (i.e., dinero y valores relativos a la Aspiración Económica), ya sea dentro de la profesión bomberil o por fuera de ella.

Al indagar la existencia de diferencias entre Bomberos Voluntarios y Remunerados, los remunerados presentan puntuaciones más elevadas en relación a las Aspiraciones Económicas; esto indica la presencia de un interés mayor asociado al logro de independencia financiera y bienes materiales que aporten mejoras a las condiciones de la vida personal (Moreno \& Marcaccio, 2012). No obstante, el mismo grupo evidencia puntuaciones más elevadas en el factor Desmotivación. Se observa entonces que el grupo de Bomberos Remunerados se encuentra más desmotivado que el grupo de Bomberos Voluntarios, pese a recibir una recompensa extrínseca (i.e., dinero) por el desarrollo de su actividad. Dicha observación, lleva a considerar el Efecto de Sobrejustificación de Hipótesis desarrollado por Lepper, Greene y Nisbett (1973), el cual afirma que los refuerzos o incentivos externos (i.e., recompensas extrínsecas) accionan negativamente, reduciendo la motivación intrínseca. Dicho de otro modo, Navarro y Quijano (2003) proponen que con frecuencia se cree que el comportamiento humano puede ser controlado mediante la aplicación de un refuerzo adecuado; dicha creencia cae cuando esas mismas recompensas comienzan a generar desmotivación en sujetos que anteriormente habían ejecutado sus tareas con niveles elevados de motivación intrínseca. No obstante, siguiendo los criterios de Cohen (1988) para la interpretación de los tamaños de efecto, vale reconocer que la baja magnitud del efecto obtenida relativiza la interpretación de las diferencias entre ambos grupos de bomberos, sobre todo, en relación a sus niveles de desmotivación, lo que permite adelantar que tal observación estadística constituye una limitación del presente estudio.

Finalmente, es atinado plantear una interrogante: $¿$ Se evidencia, en ambos grupos de bomberos, las mismas oportunidades para expresar sus motivos y valores en forma activa? Las asociaciones de Bomberos Voluntarios en la Ciudad de Buenos Aires dan cuenta de una serie de actividades mediante las cuales brindan diferentes servicios de ayuda social a la comunidad, las mismas son promocionadas en los diferentes cuarteles como así también en sus sitios web oficiales. Dichas actividades, sin lugar a dudas, propician un encuentro entre quien ejerce la profesión de bombero y el ciudadano, promueven una instancia en la cual la comunidad bomberil se encuentra más cercana a la sociedad, pudiendo manifestar sus motivos y valores de forma activa, sin necesidad de hacerlo únicamente ante situaciones de emergencia o desastre. En cambio, los Bomberos Remunerados, poseen un intermediario, alguien que oficia de mediador entre el bombero y la comunidad, es decir, la institución. Probablemente la figura normativa de la institución, imparta cierta distancia con la ciudadanía, y obstaculice la expresión de motivos y valores, por brindar restringidamente un ambiente laboral adecuado para la satisfacción de necesidades de autonomía, competencia y de relación.

\section{Limitaciones y recomendaciones}

Como se ha mencionado, esta investigación ha contado con la participación de 25 mujeres sobre un total de 130 bomberos encuestados. Al presentar dicha disparidad submuestral, no se han podido realizar 
análisis estadísticos que impliquen la segmentación de la muestra en función del género. Asimismo, dicha situación invita a reflexionar acerca de la participación de la mujer en espacios vinculados con las diferentes Fuerzas (e.g., de Seguridad, Armadas, etc.). En los últimos años, y como resultado de una deconstrucción social que se ha ido extendiendo por varios países de América Latina, se ha logrado la visibilización de la mujer en diferentes espacios a los cuales antes no podía acceder.

Por otro lado, resulta muy complejo obtener información actualizada (i.e., dotación de los diferentes cuarteles, número de intervenciones, entre otros) sobre la población estudiada, ya que las instituciones pertenecientes al propio cuerpo de bomberos que recolectan los datos, los difunden escasamente en forma pública. Esta precariedad de la información podría generar dificultades al momento de elaborar estrategias que aborden las diferentes problemáticas que puedan surgir en relación a la comunidad bomberil.

A lo largo de este trabajo se ha desarrollado la importancia de las variables estudiadas, por lo que resulta recomendable la realización de estudios vinculantes que continúen analizando la Motivación y los Valores Relativos al Trabajo en diferentes grupos de voluntarios y personal remunerado. En tal sentido, sería importante seleccionar muestras de mayor tamaño que incrementen las magnitudes de efecto alcanzadas, mejorando la significación e implicancias teóricas y empíricas de los resultados. A su vez, también es interesante que se lleven a cabo investigaciones que releven muestras que incluyan mayor cantidad de mujeres pertenecientes a cuarteles de bomberos, y otras Fuerzas, y de esta forma enriquecer los resultados obtenidos desde una perspectiva que involucre la actuación de la mujer frente a situaciones de riesgo y emergencia.

\section{Conflicto de intereses}

Durante el desarrollo de la investigación, no existieron conflictos de interés de ninguna índole (e.g., de investigación, morales, económicos, laborales, etc.) al momento de llevar a cabo la evaluación de los participantes, siendo el consentimiento informado mencionado un instrumento aplicado para legitimar la responsabilidad de la actuación individual.

\section{Responsabilidad ética}

Las cuestiones éticas consideradas al momento de realizar la presente investigación, específicamente en lo atinente a la evaluación psicométrica de humanos, se ajustan a los criterios establecidos por la Asociación Americana de Psicología (American Psychological Association, 1995; Richaud, 2007), en relación al consentimiento informado, al manejo de los datos recopilados de la batería de test psicológicos, etc.

Se aclaró a los participantes explícitamente, i.e., en la consigna de administración de test, que los datos no serían individualizados ni difundidos en la institución a fin de salvaguardar la identidad personal de cada uno, y que los resultados hallados serían utilizados únicamente para fines académicos-científicos.

\section{Agradecimientos}

Agradecemos especialmente a todos los bomberos que han participado en la investigación de forma desinteresada, contestando cada uno de los cuestionarios administrados.

\section{Referencias}

Alchourrón de Paladini, M., Daverio, P. A., Moreno, E. M., \& Piattini-Montero, J. (2011). Promoción de la resiliencia en adolescentes de una escuela semirural. En A. Mellillo \& E. N. Suárez Ojeda (comps.), Resiliencia: descubriendo las propias fortalezas (pp. 169-184). Buenos Aires: Paidós.

American Psychological Association (1995). Ethical conflicts in psychology. Washington: American Psychological Association.

Arce, M. (2015). Los servicios de emergencia en Argentina: el caso de los Bomberos de la Policía Federal destacados en la Ciudad Autónoma de 
Buenos Aires. (Tesis de Maestría). Universidad de San Andrés y Diario Clarín. Buenos Aires, Argentina.

Baard, P. P., Deci, E. L., \& Ryan, R. M. (2004). Intrinsic Need Satisfaction: A Motivational Basis of Performance and Weil Being in Two Work Settings. Journal of applied social psychology, 34(10), 20452068. doi: 10.1111/j.1559-1816.2004.tb02690.x

Bandura, A. (1975). The ethics and social purposes of behavior modification. In C. M. Franks \& G. T. Wilson (Eds.), Annual review of behavior therapy theory and practice (Vol. 3). New York: Brunner/Mazel.

Bandura, A. (1999). (Ed.). Auto-Eficacia: Cómo afrontamos los cambios de la Sociedad actual. Bilbao: Desclée de Brouwer.

Bandura, A. (2002). Social cognitive theory in cultural context. Applied Psychology: An International Review, 51, 269-290. doi: 10.1111/1464-0597. 00092

Bass, B. (1990). Handbook of leadership: a survey of theory and research. New York: Free Press.

Battistelli, A., Galletta, M., Odoardi, C., Núñez, J., \& Ntalianis, F. (2015). Proposal for a Version of MWMS across Mediterranean Countries. A Validation Study in Greece, Italy, and Spain. European Journal of Psychological Assessment, 33(2), 104-115. doi: 10.1027/1015-5759/a000277

Calandrón, S., \& Galar, S. (Diciembre, 2014). «La tragedia de Barracas». Elaboración pública de la muerte y el heroísmo en la figura de bomberos. Trabajo presentado en VIII Jornadas de Sociología de la UNLP. La Plata, Argentina.

Castro, A. (2005). Técnicas de evaluación psicológica en los ámbitos militares. Buenos Aires: Paidós.

Casullo, M. M., \& Castro, A. (2004). Valores humanos y contextos en población civil y militar. Acción Psicológica (UNED), 3(1), 21-30. doi: 10.5944/ ap.3.1.497

Chacón, F., \& Dávila, M. C. (2001). Diferencias en el perfil motivacional de voluntarios ecologistas y socio-asistenciales. Intervención Psicosocial, 10(2), 137-153.
Cohen, J. (1988). Statistical power analysis for the behavioral sciences (2. Auflage). Hillsdale, NJ: Erlbaum.

Cohen, J. (1992). A power primer. Psychological Bulletin, 112(1), 155-159. doi: 10.1037/00332909.112.1.155

Costa, P. T. Jr., \& McCrae, R. R. (1992). Revised NEO personality inventory (NEO-PI- R) and the NEO fivefactor inventory (NEO-FFI) professional manual. Odessa, FL: Psychological Assessment Resources.

Deci, E. L., \& Ryan, R. M. (1995). Human autonomy: The basis for true self-esteem. En: M. Kemis (Ed.), Efficacy, agency, and self-esteem (31-49). New York: Plenum.

Depaula, P. D. (2012). Predictores globales de la performance de estudiantes militares. Revista Ciencias Psicológicas, 6(2),135-148.

Depaula, P. D., \& Azzollini, S. C. (2012). Inteligencia cultural, valores y motivación para el aprendizaje en estudiantes militares argentinos. Revista de Psicología, 30(1), 75-102.

Diener, E., Emmons, R. A., Larson, R. J., \& Griffin, S. (1985). The Satisfaction With Life Scale. Journal of Personality Assessment, 49, 71-75.

Elshaug, C., \& Metzer, J. (2001). Personality Attributes of Volunteers and Paid Workers Engaged in Similar Occupational Tasks. The Journal of Social Psychology, 141(6), 752-763. doi: 10.1080/002 24540109600586

Gagné, M., \& Deci, E. L. (2005). Self determination theory and work motivation. Journal of Organizational behavior, 26(4), 331-362. doi: 10.10 02/job.322

Gagné, M., Forest, J., Gilbert, M. H., Aubé, C., Morin, E., \& Malorni, A. (2010). The Motivation at Work Scale: Validation evidence in two languages. Educational and Psychological Measurement, 70(4), 628-646. doi: 10.1177/0013164409355698

Gagné, M., Forest, J., Vansteenkiste, M., Crevier-Braud, L., Van den Broeck, A., Aspeli, A. K., ..., Nuñez, J. L. (2012). Validation evidence in ten languages for the revised motivation at work scale. Cross-cultural 
evidence for self-determination theory applied to the work domain. Manuscrito enviado.

Gagné, M., Forest, J., Vansteenkiste, M., Crevier-Braud, L., Van den Broeck, A., Aspeli, A. K., ... Westbye, C. (2014). The multidimensional work motivation scale: Validation evidence in seven languages and nine countries. European Journal of Work and Organizational Psychology, 24(2), 178-196. doi: 10.1080/1359432X.2013.877892

Gastañaduy-Fiestas, K. G. (2013). Motivación Intrínseca, Extrínseca y Bienestar Psicológico en Trabajadores Remunerados y Voluntarios. (Tesis de grado). Pontificia Universidad Católica del Perú. Lima, Perú.

Gutiérrez-Medina, S., Cuenca-Gómez, D., \& Álvarez-de Toledo, O. (2008). ¿Por qué quiero ser médico? Educación Médica, 11(1), 51-56.

Hernández, V. (2004). Expectativas vocacionales de resultados en los estudiantes de secundaria. Revista de Investigación Educativa, 22(1), 89-112.

Hernández, R., Fernández, C. \& Baptista, P. (2006). Metodología de la investigación. México: McGrawHill.

Lepper, M. P., Greene, D., \& Nisbett, R. E. (1973). Undermining children's Intrinsic interest with extrinsic reward: A test of the «over justification» hypothesis. Journal of Personality and Social Psychology, 28(1), 129-137. doi: 10.1037/h0035519

Locke, H. J., \& Wallace, K. M. (1959). Short maritaladjustment and prediction tests: Their reliability and validity. Marriage and Family Living, 21, 251-255. doi: $10.2307 / 348022$

Melillo, A. (2011). Resiliencia y educación. En A. Mellillo \& E. N. Suárez Ojeda (comps.), Resiliencia: descubriendo las propias fortalezas (pp. 123-144). Buenos Aires: Paidós.

Meyer, G. J., Finn, S. E., Eyde, L. D., Kay, G. G., Moreland, K. L., Dies, R. R., ... Reed, G. M. (2001). Psychological testing and psychological assessment: A review of evidence and issues. American Psychologist, 56(2), 128-165. doi: 10.1037/0003066X.56.2.128

Moreno, J. E., \& Marcaccio, A. (2012). Escala de valores relativos al trabajo. Propiedades psicométricas de una versión en castellano revisada. Praxis. Revista de Psicología, 14(22), 65-78.

Moreno, J. E., \& Marcaccio, A. (2014). Perfiles profesionales y valores relativos al trabajo. Ciencias Psicológicas, 8(2), 129-138.

Moreno, J. E., \& Schmidt, A. (2013). Motivaciones altruistas en la elección vocacional-ocupacional. Recuperado de www.enduc.org.ar/enduc7/trabajos/t36c00.docx

Nader, M., \& Castro Solano, A. (2007). Influencia de los valores sobre los estilos de liderazgo: Un análisis según el modelo de liderazgo transformacionaltransaccional de Bass. Universitas Psychologica, 6(3), 689-698.

Natenzon, C. E., \& González, S. G. (2010). Riesgo, vulnerabilidad social y construcción de indicadores. Aplicaciones para Argentina. En M. Arroyo \& P. Zusman (Eds.), Argentina e Brasil: posibilidades e obstáculos no proceso de integração territorial [Argentina y Brasil: posibilidades y obstáculos en el proceso de integración territorial], (pp. 195-217). São Paulo: Humanitas.

Navarro J., \& Quijano S. D. (2003). Dinámica no lineal en la motivación en el trabajo: propuesta de un modelo y resultados preliminares. Psicothema, 4(15), 643-649.

Peñacoba-Puente, C., Díaz-Gutiérrez, L., Goiri-Pueyo, E., \& Vega-López, R. (2000). Estrategias de afrontamiento ante situaciones de estrés: Un análisis comparativo entre bomberos con y sin experiencia. Revista de Psicología del Trabajo y de las Organizaciones, 16(3), 341-356. Recuperado de http://www.redalyc.org/articulo. oa?id=231317662006

Porto, J. B., \& Pilati, R. (2010). Escala Revisada de Valores Relativos ao Trabalho: EVT-R. Psicologia: Refexãoe Crítica, 23(1), 73-82. doi: 10.1590/S0102-79722 010000100010

Porto, J. B., \& Tamayo, A. (2003). Escala de Valores Relativos ao Trabalho - EVT. Psicologia: Teoria e Pesquisa, 19(2), 145-152. doi: 10.1590/S010237722003000200006

Ramirez, M., Harland, K., Frederick, M., Shepherd, R., Wong, M., \& Cavanaugh, J. E. (2013). Listen protect connect for traumatized schoolchildren: a pilot study 
of psychological first aid. BMC Psychology, 26(1), $1-9$.

Richaud, M. C. (2007). La ética en la investigación psicológica. Enfoques, 19(1-2), 5-18.

Ryan, R. M. (1995). Psychological needs and the facilitation of intrinsic motivation, social development and well being. American Psychologist, 63, 397-427.

Ryan, R., \& Deci, E. (2000). La teoría de la autodeterminación y la facilitación de la motivación intrínseca, el desarrollo social y el bienestar. American Psychologist, 55(1), 68-78. doi: 10.1037110003066X.55.1.68

Schwartz, S. (1994). Are there universal aspects in the structure and content of human values? Journal of Social Issues, 50, 19-45. doi: 10.1111/j.1540-4560. 1994.tb01196.x

Schwartz, S. (2001). ¿Existen aspectos universales en la estructura y contenido de los valores humanos? En M. Ros \& V. Gouveia (Eds.), Psicología Social de los valores humanos (pp. 53-76). Madrid: Biblioteca Nueva.

Schwartz, S. (2005). Basic Human Values: Their content and structure across countries. In A. Tamayo \& J. Porto (Eds.), Valores y trabalho [Values and work]. Brasilia: Editora Universidade de Brasilia.

Schwartz, S., Melech, G., Lehman, A., Burgess, S., Harris, M., \& Owens, V. (2001). Extending the cross-cultural validity of the theory of basic human values with a different method of measurement. Journal of Cross Cultural Psychology, 32(5), 519-542. doi: 10.1177/ 0022022101032005001

Scrymgeour, G. C., Smith, L., \& Paton, D. (2016). Exploring the Demandson Nurses Working in Health Care Facilities During a Large-Scale Natural Disaster: Oftenan Invisible Role Within a Highly Visible Event. SAGE Open, April-June, 1-7. doi: 10.1177/2158244 016655587

Soria, M., Guerra, M., Giménez, I., \& Escanero, J. F. (2006). La decisión de estudiar medicina: Características. Educación Médica, 9(2), 91-97.

Traut, C. A., Larsen, R., \& Feimer, S. H. (2000). Hangingonor fading out? Job satisfaction and thelongtermworker. Public Personnel Management, 29, 343-351. doi: 10.1177/009102600002900304

Uriarte-Arciniega, J. (2010). Psicología Positiva y sus dificultades. La resiliencia comunitaria en situaciones catastróficas y de emergencia. International Journal of Developmental and Educational Psychology, 1(1), 687-693.

Wagner, S. L., \& O’Neill, M. (2012). Job, Life, and Relationship Satisfaction for Paid-Professional Firefighters. Journal of Loss and Trauma, 17, 423438. doi: 10.1080/15325024.2011.650129

Zubieta, E. M., Filippi, G., \& Báez, X. (2007). Valores y creencias asociadas al trabajo en estudiantes universitarios de Argentina y Chile. SUMMA Psicológica UST, 4(2), 81-98.

\section{Florencia Rocío Piedimonte}

Universidad Abierta Interamericana (Graduada)

Licenciada en Psicología. Desarrollo de estudios complementarios en población de bomberos para la elaboración de tesina de grado.

ORCID: 0000-0003-2831-7897

florencia.piedimonte@gmail.com

Pablo Domingo Depaula

Consejo Nacional de Investigaciones Científicas y Técnicas (Becario Postdoctoral).

Doctor en Psicología de la Universidad de Buenos Aires, Magister en Psicología Organizacional con orientación Gerencial de la Universidad Abierta Interamericana, y especialista en Psicología Social con orientación en Emergencia y Catástrofe del Colegio de Psicólogos de la provincia de Buenos Aires. Desarrollo de estudios que abordan la construcción de un perfil óptimo de voluntarios en primeros auxilios psicológicos frente a situaciones de desastre y catástrofe.

ORCID: 0000-0002-5610-9131

Autor corresponsal: lic_pablodepaula@yahoo.com.ar 